\section{How to implement endurance exercise training in sickle cell disease}

A previous study demonstrated the safety and beneficial effects of moderate-intensity endurance exercise training in patients with sickle cell disease (SCD). However, this previous pilot study had several limitations. The most important constraint was the need for repeated measurements of blood lactate concentration in order to adjust training intensity. This approach considerably hinders implementation and dissemination of endurance exercise training in the general population of SCD patients. In the present study, we tested heart rate as a possible surrogate for adjustment of training intensity. Patients $(\mathrm{n}=15)$ successively performed an initial submaximal incremental test (SIT1), 30-min moderateintensity cycling exercises three times a week for 8 weeks, and finally another SIT (SIT2). Heart rate (HR) and blood lactate concentration ([lactate $]_{b}$ ) were recorded every minute during both SIT and once during training sessions. [lactate $]_{b}$ and $\mathrm{HR}$ values measured during training concurred with the correlation obtained between [lactate $]_{b}$ and HR during the two SIT. Provided

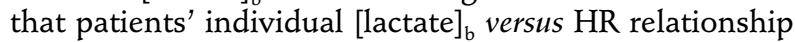
(obtained during a SIT) is known and patients are accustomed to cycling exercise, HR appears to be an acceptable surrogate to control and adjust intensity of endurance exercises.

SCD is the most frequent genetic disease worldwide. Vaso-occlusion and anemia, the two main consequences of the disease, induce a myriad of complications affecting

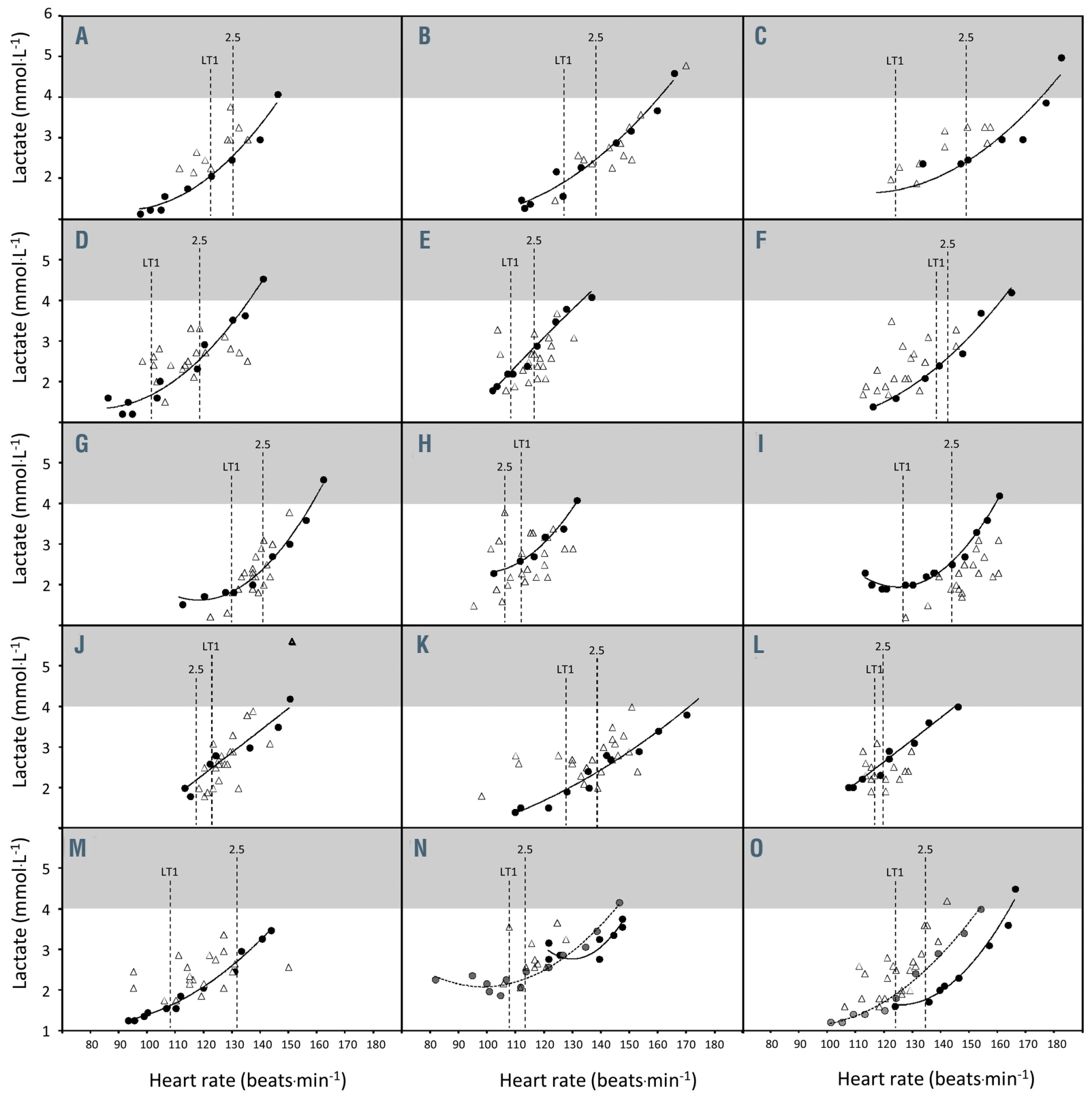

Figure 1. Relationship between blood lactate concentration and heart rate in 15 patients with sickle cell disease during exercise. The relationship between blood lactate concentration ([lactate $]_{b}$ ) versus heart rate (HR) relationship during a first submaximal incremental test (black circles) and a second one for two patients (gray circles, panels $\mathrm{N}$ and $\mathrm{O}$ ), as well as the [lactate $]_{b}-\mathrm{HR}$ pairs measured during the training sessions (open triangles). HR at the first lactate threshold (LT1) as well as at $2.5 \mathrm{mmol} . \mathrm{L}^{-1}$ are also reported (vertical dotted lines). 
almost all organs. Physical exercise was, until recently, considered a risk for SCD patients. Indeed, 31\% of vasoocclusive crises and $32 \%$ of secondary acute chest syndromes occur subsequent to exertion. ${ }^{1}$ However, recent reports provided evidence that when well-calibrated, moderate-intensity endurance-exercise training in adults with SCD without severe chronic complications seems safe, significantly improved functional capacity and is a potential novel therapeutic strategy. ${ }^{2,3}$ Although encouraging, the previous pilot study suffered from several constraints, limiting its scientific and clinical impact: the training program was relatively short, involved a limited

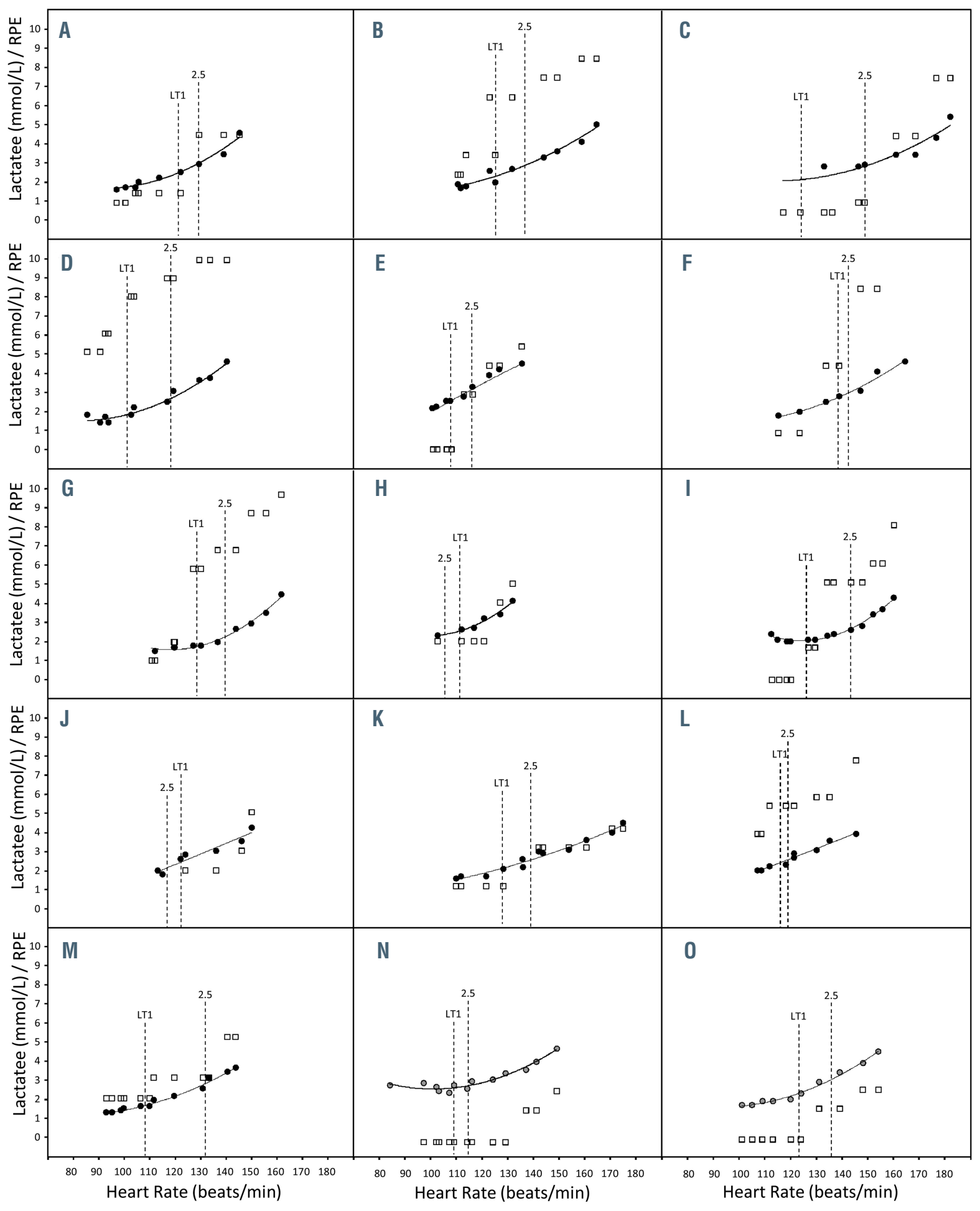

Figure 2. Relationship between blood lactate concentration, rating of perceived exertion and heart rate in 15 patients with sickle cell disease during exercise. The relationship between blood lactate concentration ([lactate $\left.]_{b}\right)$ versus heart rate (HR) relationship during a first submaximal incremental test (black circles) or a second one for two patients (gray circles, panels $\mathrm{N}$ and 0 ), as well as the rating of perceived exertion (RPE) values obtained during the same trials (open squares). HR at the first lactate threshold (LT1) as well as at $2.5 \mathrm{mmol}^{-\mathrm{L}^{-1}}$ are also reported (vertical dotted lines). 
number of patients, was performed on cycle-ergometers in a medical center under the supervision of a physician, and required repeated [lactate $]_{b}$ measurements. ${ }^{2,3}$ These limitations hinder implementation and dissemination of long-term endurance exercise training in the general population of SCD patients. The key point was therefore to find a non-invasive marker to adjust exercise intensity during endurance training and physical activity in SCD patients. In this study we proposed and tested HR as the possible surrogate (ClinicalTrials.gov, number NCT02571088).

Data from eight male (53\%) and seven female (47\%) adult SCD patients (age: $34 \pm 11$ years; height: $172 \pm 10 \mathrm{~cm}$; weight: $66 \pm 11 \mathrm{~kg}$ ) were analyzed. Patients successively underwent a first submaximal incremental test (SIT1), an 8 -week endurance exercise training program, and a posttraining SIT (SIT2). SIT1 and SIT2 started at 20 or $30 \mathrm{~W}$ and increased every $2 \mathrm{~min}$ by 10 or $15 \mathrm{~W}$ for females and males, respectively. $\mathrm{HR}$ and [lactate $]_{b}$ were recorded every minute. HR was derived from a 12-lead electrocardiogram (ErgoCard, Medisoft, Sorinnes, Belgium). For [lactate $]_{b}$, a blood drop $(10 \mu \mathrm{L})$ was taken from the earlobe and tested extemporaneously within 15-20 s (Lactate Scout+, EKF diagnostics, Cardiff, UK). Exercise was stopped as soon as [lactate $]_{b}$ was $\geq 4 \mathrm{mmol.L^{-1 }}$. HR was determined at the first lactate threshold (LT1) and at $2.5 \mathrm{mmol} . \mathrm{L}^{-1}$ of [lactate $]_{\mathrm{b}}$. Three times a week, patients performed 30-min moderate-intensity cycling exercises, for 8 weeks. [Lactate $]_{b}$ and HR were measured during the training sessions. Data are presented as the mean \pm standard deviation. HR and [lactate $]_{\mathrm{b}}$ obtained during SIT were correlated using polynomial regressions. Standard errors of the estimate (SEE) to the regression line were calculated.

Figure 1 reports [lactate $]_{\mathrm{b}}$ versus $\mathrm{HR}$ curves (circles) obtained during SIT1 for the 15 patients (A to O) and also during SIT2 for patients $\mathrm{N}$ and $\mathrm{O}$. The mean $\mathrm{R}^{2}$ of the [lactate $]_{\mathrm{b}}$ versus $\mathrm{HR}$ polynomial regressions was $0.954 \pm 0.056$. The mean SEE value to the polynomial

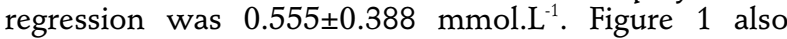
reports [lactate $]_{\mathrm{b}}$ and the associated HR measured during the training sessions (triangles). The mean SEE value of [lactate $]_{b}$ during training to the polynomial regression was $0.714 \pm 0.047 \mathrm{mmol} . \mathrm{L}^{-1}$. During training, no patient who had a HR in the range between LT1 and

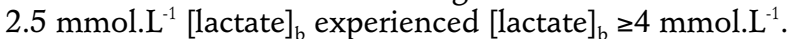

The present study aimed to find a surrogate for [lactate $]_{b}$ to implement and widely disseminate endurance exercise training among SCD patients. If the conditioning strategy is to target an exercise intensity low enough to be safe and elevated enough to induce adaptations, an exercise intensity between LT1 and $2.5 \mathrm{mmol}^{-1}$ [lactate $]_{\mathrm{b}}$ might be targeted. ${ }^{4}$ A safety cutoff should be set at $4 \mathrm{mmol} . \mathrm{L}^{-1}$ since above this value blood lactate accumulates abruptly and associated acidosis may develop rapidly, especially in SCD. ${ }^{4}$ We paid particular attention to prevent lactate accumulation-associated acidosis, which constitutes a potentially major exercise-related triggering factor of $\mathrm{HbS}$ polymerization, sickling and vaso-occlusive crises. ${ }^{5}$

A first possibility to implement endurance exercise would be to use the power output corresponding to a target $[\text { lactate }]_{b}$ obtained during SIT. However, this would oblige patients to exercise on a cycle ergometer so that any other forms of physical activity would be excluded.

A second possibility would be to use HR. In 13 patients, [lactate $]_{\mathrm{b}}$ and the associated $\mathrm{HR}$ recorded during training concurred with the correlation obtained during SIT1. We noted that the two remaining patients did not become adequately accustomed to cycling during SIT1, unlike the 13 other patients who adapted rapidly. If the SIT2 data were taken into account for these two patients, the concordance was clear again (Figure 1, panels $\mathrm{N}$ and O). As a whole, all $\mathrm{HR}$ values recorded during training sessions within the LT1 and $2.5 \mathrm{mmol} . \mathrm{L}^{-1}$ window were associated with [lactate $]_{b}$ close to the target values and never above $4 \mathrm{mmol.L^{-1 }}$ (the safety cutoff).

Another possibility would have been to use patients' rate of perceived exertion (RPE). However, the sensitivity of RPE appeared (at least in our population) too limited: several patients reported similar RPE for a wide range of power outputs crossing the LT1-2.5 mmol. $\mathrm{L}^{-1}$ window (Figure 2). This large intra- and inter-individual variability may constitute a pitfall to the use of RPE as a means to manage exercise intensity precisely during training sessions. However, further studies would be necessary to confirm this point.

Taken together, the present data indicate that HR appears to be an acceptable surrogate to implement endurance exercise, as long as patients' individual [lactate $]_{b}$ versus HR relationship (obtained during SIT) is known and patients are accustomed to cycling exercise. To ensure safety and obtain benefit, an exercise intensity objectified by HR ranged between LT1 and 2.5 mmol. $\mathrm{L}^{-1}$ obtained during SIT could be used. Further prospective studies would be needed: (i) to confirm the accuracy of $\mathrm{HR}$ as a surrogate for [lactate $]_{b}$ during endurance training of SCD patients, and (ii) to determine the frequency with which SIT must be repeated to take into account the improvements/changes induced by endurance training.

Laurent A. Messonnier, ${ }^{1}$ Manon Riccetti, ${ }^{1}$ Benjamin Chatel, ${ }^{2}$ Frédéric Galactéros, ${ }^{3,4}$ Barnabas Gellen, ${ }^{5}$ Thomas Rupp, Léonard Féasson ${ }^{6,7}$ and Pablo Bartolucci, ${ }^{3,4}$

${ }^{1}$ Université Savoie Mont Blanc, Laboratoire Interuniversitaire de Biologie de la Motricité, EA7424, Chambéry; ${ }^{2}$ CellMade, Le Bourgetdu-Lac; ${ }^{3}$ Sickle Cell Referral Center, UMGGR, Hôpital Henri-

Mondor, Assistance Publique-Hôpitaux de Paris (AP-HP), Université Paris-Est Créteil (UPEC), Créteil; Institut Mondor de Recherche Biomédicale, Unité 2, INSERM, Hôpital Universitaire Henri-Mondor, Université Paris-Est Créteil (UPEC), Créteil; ${ }^{5}$ Department of Cardiac Rehabilitation, Henri-Mondor University Hospital, Assistance Publique-Hôpitaux de Paris (AP-HP), Créteil; ' ${ }^{6}$ niversité de Lyon, Université Jean Monnet, Laboratoire Interuniversitaire de Biologie de la Motricité, EA 7424, Saint-Etienne and ${ }^{7}$ Unité de Myologie, Service de Physiologie Clinique et de l'Exercice, Hôpital Universitaire de Saint-Etienne, Saint-Etienne, France

Correspondence:

LAURENT A.MESSONNIER - laurent.messonnier@univ-smb.fr doi:10.3324/haematol.2020.267047

Received: July 16, 2020.

Accepted: August 26, 2020.

Pre-published: August 27, 2020

Disclosures: $L A M$ has received gifts and consulting fees from Addmedica and bluebirdbio, respectively; $P B$ has received grants and/or consulting fees from Addmedica, bluebirdbio, Emmaus, Fondation Fabre, GBT, Hermanext, Novartis and Roche. These gifts, fees and grants are not relevant to the submitted work.

Contributions: $L A M, F G, L F$ and $P B$ designed and conducted this study; MR and BC analyzed data; MR created the figures; $L A M$ wrote the first draft of the article. All authors critically reviewed the draft and approved the final version for publication.

Funding: this work was supported in part by research funding from "l'association l'ar'mony". 


\section{References}

1. Bartolucci P, Habibi A, Khellaf M, et al. Score predicting acute chest syndrome during vaso-occlusive crises in adult sickle-cell disease patients. EBioMedicine. 2016;10:305-311.

2. Gellen B, Messonnier LA, Galacteros F, et al. Moderate-intensity endurance-exercise training in patients with sickle-cell disease without severe chronic complications (exdre): An open-label randomised controlled trial. Lancet Haematol. 2018;5(11):e554-e562.
3. Merlet AN, Messonnier LA, Coudy-Gandilhon C, et al. Beneficial effects of endurance exercise training on skeletal muscle microvasculature in sickle cell disease patients. Blood. 2019;134(25):22332241

4. Messonnier LA, Gellen B, Lacroix R, et al. Physiological evaluation for endurance exercise prescription in sickle cell disease. Med Sci Sports Exerc. 2019;51(9):1795-1801.

5. Chatel B, Messonnier LA, Bendahan D. Do we have to consider acidosis induced by exercise as deleterious in sickle cell disease? Exp Physiol. 2018;103(9):1213-1220. 